\title{
Dietary support in insulin resistance: An overview of current scientific reports
}

\author{
Katarzyna Daria Gołąbek ${ }^{A-F}$, Bożena Regulska-Ilow ${ }^{A, C-F}$ \\ Department of Dietetics, Faculty of Health Sciences, Wroclaw Medical University, Poland \\ A - research concept and design; B - collection and/or assembly of data; $C$ - data analysis and interpretation; \\ $\mathrm{D}$ - writing the article; $\mathrm{E}$ - critical revision of the article; $\mathrm{F}$ - final approval of the article
}

Address for correspondence

Katarzyna Gołąbek

E-mail: katarzyna.golabek@umed.wroc.pl

Funding sources

None declared

Conflict of interest

None declared

Received on June 3, 2018

Reviewed on March 15, 2019

Accepted on June 11, 2019

Published online on November 18, 2019

Cite as

Gołąbek K, Regulska-llow B. Dietary support in insulin resistance: An overview of current scientific reports. Adv Clin Exp Med. 2019;28(11):1577-1585. doi:10.17219/acem/109976

DOI

10.17219/acem/109976

\section{Copyright}

Copyright by Author(s)

This is an article distributed under the terms of the

Creative Commons Attribution Non-Commercial License

(http://creativecommons.org/licenses/by-nc-nd/4.0/)

\begin{abstract}
Over the past 30 years, a significant increase in the prevalence of insulin resistance (IR) has been observed. It is associated with more frequent occurrence of impaired glucose tolerance, diabetes, excessive weight, cardiovascular diseases, and endocrine disorders. The results of current studies do not indicate a necessity to exclude dairy products from the diet of insulin-resistant individuals. In addition, it has been found that moderate amounts of alcohol as part of a balanced, low-energy diet do not have a negative effect on insulin sensitivity. The authors of recent studies emphasize the importance of reducing the intake of simple sugars, especially from sweet drinks, sweets and excessive fruit juice consuption. Many studies have demonstrated the beneficial effects of consuming complex, low-glycemic-index carbohydrates that are rich in dietary fiber. An insulin-resistant patient's diet should be rich in whole grains and high amounts of non-starchy vegetables and raw fruit. The beneficial effect of the Dietary Approaches to Stop Hypertension (DASH diet) and the Mediterranean diet has been confirmed. The positive correlation between low-carbohydrate and very-low-carbohydrate diets requires confirmation in long-term studies with the participation of insulinresistant patients. Research shows the benefits of increased calorific intake during the first half of the day, especially from a high-energy and low-glycemic-index breakfast. Furthermore, many researchers indicate that slow and mindful eating is a significant component of an appropriate diet for insulin-resistant individuals.
\end{abstract}

Key words: insulin resistance, diet, glycemic index 
Insulin resistance (IR) is an important issue in modern medicine. Over the past 30 years, a significant increase in the detection of IR has been observed. ${ }^{1}$ Insulin resistance is associated with lower insulin sensitivity of the body tissues. It may be due to abnormal functioning and signaling of insulin receptors, an excessively high level of insulin-binding antibodies or an abnormal insulin molecule structure. ${ }^{2}$ This condition is associated with an increased incidence of hyperinsulinemia, impaired glucose tolerance and diabetes. ${ }^{3}$

The frequency of IR depends on age,${ }^{4}$ body weight, ${ }^{3}$ sex, ${ }^{5}$ genetic predisposition, ${ }^{6}$ physical activity, and lifestyle. ${ }^{7}$ In addition, it has been shown that it can be associated with stress and overstimulation of the sympathetic nervous system. ${ }^{8}$ The occurrence and development of IR are strongly correlated with inflammation. ${ }^{9}$

The prevalence of IR is estimated at between 10-30\%, depending on the study population. In a study involving 1,500 adolescents aged 13-18, IR (defined as a Homeostatic Model Assessment of Insulin Resistance (HOMA-IR) >4) was diagnosed in approx. $10.8 \%$ of girls and $14.3 \%$ of boys. The proportion increased to $29.1 \%$ and $44 \%$, respectively, in the obese group, and decreased to $4.7 \%$ and $7.2 \%$, respectively, in the normal body weight group. ${ }^{10}$ In a study on Asian adults over 30 years of age, its incidence was estimated at $21.5 \%$ in women (HOMA-IR $\geq 1.64$ ) and $25.1 \%$ in men (HOMA-IR $\geq 1.56) .{ }^{11}$ In one European population (Estonian adults), the percentage of insulin-resistant individuals (HOMA-IR $\geq 1.92$ ) amounted to approx. $12.4 \%$ in people without metabolic syndrome and $58.8 \%$ in people with it. ${ }^{12}$

Insulin resistance is strongly related to overweight and obesity. ${ }^{3}$ In addition, it correlates with a higher prevalence of dyslipidemia, cardiovascular disease $\mathrm{e}^{13}$ and endocrine disorders such as polycystic ovary syndrome (PCOS), hypercortisolemia and hyperprolactinemia. ${ }^{2}$

Lifestyle, eating habits and physical activity are important factors that can sustain or increase the risk of IR. A well-adjusted, balanced diet and regular meal frequency may improve glucose/insulin homeostasis.

\section{Alcohol consumption and insulin sensitivity}

Even though a significant reduction or exclusion of alcohol is recommended to overweight and obese patients with metabolic complications, studies on IR have not shown any clear negative influence of alcohol consumption on the course of the disease. Some of them indicate that moderate consumption of ethanol has a positive impact on the parameters related to insulin homeostasis. In a study involving 1,542 Japanese men aged 46-58 years, it was observed that daily consumption of $30 \mathrm{~g}$ or more of ethanol was related to a $40 \%$ decrease in the risk of IR (defined as HOMA-IR $\geq 1.81$ and fasting plasma insulin levels $\geq 50 \mathrm{pmol} / \mathrm{L}) .{ }^{14}$ In another study with 249 patients with IR (HOMA-IR $\geq 2.5$ ), the authors observed a negative correlation between alcohol consumption and HOMAIR values in all alcohol-consumption groups, i.e., light (40-140 g/week), moderate (140-280 g/week) and heavy (>280 g/week)..$^{15}$

However, the authors of meta-analysis of 14 studies found no significant impact of moderate alcohol consumption (i.e., below $40 \mathrm{~mL} /$ day) on IR. Some results suggest a possible positive influence of moderate consumption only in women. ${ }^{16}$

A 20-year analysis of changes in insulin sensitivity among people aged 18-30 years, based on the quantitative insulin sensitivity check index (QUICKI) showed that alcohol consumption did not affect insulin sensitivity. However, smoking was significantly associated with its deterioration. ${ }^{17}$

Some authors note that abstinence from alcohol may increase insulin sensitivity in particular organs. In a study involving 8 non-obese men with elevated fasting glucose levels who consumed approx. $32.1 \mathrm{~g}( \pm 20 \mathrm{~g})$ of alcohol per day, it was shown that refraining from alcohol for a week increased the insulin sensitivity of the liver. However, this was not related to muscle insulin sensitivity. It appears that reduced insulin sensitivity of the liver is associated with a higher percentage of visceral adipose tissue, correlating with metabolic disorders, aggravated inflammation and IR in patients with type 2 diabetes. These observations need confirmation in a larger study group. ${ }^{18}$

\section{Milk, dairy products and IR}

In conjunction with theories regarding insulin index or insulinotropic effect of consuming milk and dairy products, ${ }^{19}$ the merits of their consumption in a group of patients with carbohydrate metabolism disorders and IR is often considered. In a study with 78 metabolically obese but normal weight (MONW) men and 154 nonobese metabolically healthy men, daily dairy consumption was associated with a $56 \%$ lower risk of MONW, which was defined by HOMA-IR $\geq 2$.48. Furthermore, more frequent consumption of dairy products, i.e., over 1.4 portions/day compared to under 0.8 portions/day, was associated with lower HOMA-IR values. ${ }^{20}$ Similar results were obtained in a randomized study involving 23 healthy subjects. It was observed that consumption of more than 4 portions of lowfat dairy products per day for 12 months was associated with a 9\% decrease in fasting insulin level and an 11\% decrease in HOMA-IR values, compared to people consuming fewer than 2 portions per day. ${ }^{21}$ In another study evaluating milk intake alone in a group of 19 people, daily consumption of approx. 1.5 L of UHT or pasteurized milk for 21 days in a row did not influence HOMA-IR values. ${ }^{22}$

However, not all research results confirm a neutral or positive effect of dairy consumption. In a study involving 47 patients with excessive body weight, following 
a high-dairy diet (4-6 portions of dairy products daily, e.g., $250 \mathrm{~mL}$ of milk, $200 \mathrm{~g}$ of yogurt, $40 \mathrm{~g}$ of hard cheese, $120 \mathrm{~g}$ of ricotta) for 4 weeks, it was associated with higher fasting insulin levels than after 4 weeks of a high-red meat, low-dairy diet (less than 1 portion of dairy products daily). Among women following a high-dairy diet, a decrease in insulin sensitivity assessed with HOMA-IR and the Matsuda insulin index (Matsuda-IS) was observed. ${ }^{23}$

These studies do not indicate that it is necessary to exclude dairy products in order to increase insulin sensitivity. Assessments of the beneficial effect of dairy consumption on the occurrence and severity of IR require confirmation in studies with larger research groups.

\section{The relationship between consumption of high-fiber products and occurrence of IR}

Fruit and vegetables are at the base of the food pyramid and are a source of essential vitamins and minerals. However, not all studies confirm that consuming them has a clearly positive effect on IR. It seems that the quality of carbohydrates and total daily dietary fiber intake play a more important role in terms of this disorder.

In a study involving 89 overweight patients at risk of cardiovascular disease, no changes in insulin response (measured with the metabolic clamp technique) were observed after a 12-week period of increased fruit and vegetable consumption. No significant differences were found for 2 , 4 or 7 additional servings of fruit and vegetables per day. ${ }^{24}$ Similarly, in a study involving 6,000 Indian citizens, no impact of grain legumes consumption on the level of insulin sensitivity was found. ${ }^{25}$ Moreover, in a group of 8-to-10year-olds, the number of portions of fruit and vegetables was not related to the values of IR indices such as HOMAIR and Matsuda-IS. ${ }^{26}$

However, in another study based on the Food Frequency Questionnaire (FFQ) and 3-day food records with 24-hour recall in 250 Koreans aged 20-81 years, it was observed that there is a negative correlation between green and yellow vegetable consumption and fasting insulin level as well as HOMA-IR values. ${ }^{27}$

The proportion of the total weight of consumed vegetables and fruits (excluding juices) on the one hand and other consumed food on the other should be taken into account when planning a diet for a person with impaired glucose/insulin homeostasis. Cavallo et al. found that lower fruit and vegetable intake, compared with that of other foods consumed, were associated with lower fasting insulin levels. ${ }^{28}$

A meta-analysis of 12 studies concerning the impact of fruit juice consumption (about 50-500 mL per day) on fasting glucose and insulin level and HOMA-IR values did not show any significant relation between this habit and fasting glucose and insulin levels. Only 3 studies confirmed a significant correlation between juice consumption and increased HOMA-IR values. However, this result could be related to high heterogeneity of the study group. A random effect model analysis did not confirm the significance of the correlation between juice consumption and increased HOMA-IR values. ${ }^{29}$ Similar results were obtained by Simpson et al. ${ }^{30}$ However, another study, involving children at the age of 12-17 years, found a relationship between more frequent consumption of fruit juices and higher HOMA-IR values among the female participants. ${ }^{31}$

Silveira et al. observed a positive effect of consuming approx. $750 \mathrm{~mL}$ of antioxidant-rich blood orange juice in a group of insulin-resistant individuals (HOMAIR > 2.71) with normal body mass index (BMI) values. ${ }^{32}$ After 8 weeks of juice consumption, fasting insulin levels were reduced by $25 \%$ and HOMA-IR values were reduced by $28 \%$ as compared to the results from the beginning of the study. This correlation was not observed among the participants with excessive body weight. It seems that the positive effect could be the result of a decrease in inflammation associated with the consumption of the antioxidants contained in the juice.

Although the influence of fruit juice consumption on IR is unclear, it has been confirmed that whole fruit consumption may be associated with lower HOMA-IR values. ${ }^{28}$

Dietary fiber from whole grains and vegetables is an important component of the diet of insulin-resistant individuals. Many studies showed a positive correlation between its consumption and the values of indicators used to assess this condition. In a study involving 1,494 middle-aged Finns, based on 4-day food intake records and biochemical parameters, it was estimated that the total intake of dietary fiber and wholegrain bread was associated with lower Matsuda-IS values, regardless of the physical activity level or waist circumference. High dietary fiber intake correlated with lower secretion of insulin. Among the female participants, it was observed that dietary fiber intake was related to the level of insulin secretion in the early phase of the oral glucose tolerance test and inversely correlated with HOMA-IR values. In addition, total fat intake below $30-35 \%$ of the energy from the diet (E) and a high intake of saturated fatty acids (SFA) were positively associated with HOMA-IR values and negatively with Matsuda-IS values. ${ }^{33}$ Similarly, a study of 674 children found that the total daily dietary fiber intake and the consumption of whole grains such as bread and cereals were inversely correlated with fasting insulin levels. ${ }^{34}$

A meta-analysis of 3 studies concerning the effect of complex carbohydrate consumption on insulin and glucose levels showed that eating breakfasts high in complex carbohydrates (12-21 g) was associated with better glucose and insulin responses, compared to meals with a very low content of complex carbohydrates (approx. 0.1-0.9 g), the same energy content (approx. 19-22\% of E) and similar levels of other macroelements. ${ }^{35}$ 
MacNeil et al. obtained similar results by assessing the effect of an increased supply of dietary fiber on glucose and insulin levels after breakfast in a group of 12 diabetic patients. ${ }^{36}$ In this study, it was observed that replacing $20 \mathrm{~g}$ of simple carbohydrates with complex carbohydrates in a breakfast bagel (total carbohydrate content $50 \mathrm{~g}$ ) significantly reduced glucose and insulin blood levels after the meal compared to the results after eating a product with the same total carbohydrate content but lower dietary fiber content ( $2 \mathrm{~g})$.

Results from studies investigating the influence of complex carbohydrates suggest that they have a positive impact on insulin and glucose levels. However, this topic requires further studies involving larger numbers of subjects to determine the appropriate amount of carbohydrates in the meals of insulin-resistant individuals.

\section{Sweetened drinks, sweet products and fast foods, and IR}

Sweetened drinks, juices and sweets are a major source of excess simple sugars in the diet. Reducing the intake of these sugars is a particularly important element in the treatment of insulin-resistant patients. Simple sugars increase both postprandial glycemia and insulinemia.

In a study with the participation of over 2,000 people, a significant relationship was observed between HOMA-IR index values and the frequency of consumption of sweetened drinks (estimated using the FFQ). This effect was not found in the case of artificially sweetened beverages. In addition, it was assessed that people who consumed more than 3 portions of sweetened drinks per week showed a higher risk of pre-diabetes. ${ }^{37}$

Similar observations were made by O'Connor et al. in a study of 9,700 respondents. ${ }^{38}$ Based on an FFQ containing 130 products, a positive correlation was observed between the intake of simple sugars from drinks (juices, carbonated and non-carbonated soft drinks, sugar-sweetened beverages) as well as added sugars (sugars from 100\% juices, table sugar, honey, and syrups) and the occurrence of IR. People in the $3^{\text {rd }}$ quartile of consumption of these products showed the highest HOMA-IR values.

In another study, it was estimated that higher intake of sweetened soft drinks, low-calorie soft drinks, onions, garlic, French fries, salty snacks, as well as bread and rolls made from refined flour correlated with higher HOMA-IR values. An inverse relationship was observed in relation to medium- and high-fiber cereal, jam, marmalade, honey, French dressing, vinaigrette sauce, and wholegrain bread. ${ }^{39}$

Sweets and sweet snacks are a significant problem in children's diets. In a study involving 1,912 Greek children aged 9-13 years, it was observed that increased consumption of candies, lollipops, jellybeans, fruit in sugar syrup, and salty snacks was associated with a higher incidence of IR (HOMA-IR > 3.16). ${ }^{40}$ However, in another study, involving
1,153 people aged $18-69$ years and based on a partial FFQ, it was found that HOMA-IR values and fasting insulin levels were significantly lower among regular consumers of chocolate. Among the respondents, $80 \%$ were considered regular consumers, eating about $25 \mathrm{~g}$ of chocolate per day. It was reported that daily consumption of $100 \mathrm{~g}$ of chocolate was associated with HOMA-IR index values lower by approx. 0.16 units. $^{41}$ However, the study did not assess what percentage of daily energy intake came from chocolate consumption and whether or not this consumption leads to weight gain, which seems highly significant in the context of IR.

\section{Diets used in IR}

In many studies about insulin sensitivity, besides the assessment of the impact of particular components of diet on the glucose-insulin metabolism, the effectiveness of comprehensive dietary plans on the condition of insulinresistant patients has also been evaluated. Many researchers have found that diets with modified levels of particular FA, low-glycemic-index diets, the Dietary Approaches to Stop Hypertension (DASH diet), and the Mediterranean diet have positive effects. ${ }^{42-52}$

\section{The relationship between IR and the Mediterranean diet, the DASH diet and a vegetarian diet}

An analysis of the data obtained from the patients of the Third National Health and Nutrition Examination Survey (NHANES III) showed that scores indicating the similarity of the respondents' diet to the Mediterranean diet were associated with lower BMI and waist circumference. Lower waist circumference correlated with lower glycated hemoglobin levels, fasting insulin levels and HOMA-IR index values. ${ }^{42}$

Mattei et al. compared degree of similarity of patients' diets to well-known diets such as the Mediterranean diet (using Mediterranean diet score (MeDS)), the DASH diet and the American Heart Association (AHA) diet (using AHAdiet score), and assessed their quality using the Healthy Eating Index - 2005 and the Alternative Healthy Eating index $2010 .{ }^{43}$ It was found that the Mediterranean diet had the strongest association with lower insulin levels, lower HOMA-IR values and higher levels of insulin sensitivity. A high content of homemade vegetable soups, nonprocessed home-cooked meat, oatmeal, grain legumes, fish, whole milk, and beer in the diet had the strongest association with higher insulin sensitivity.

A 24-week study evaluated the impact of following a vegetarian diet and a diabetic diet on the level of insulin sensitivity in type 2 diabetics with IR. ${ }^{44}$ In the vegetarian diet, 
$60 \%$ of E came from carbohydrates, $15 \%$ from protein and $25 \%$ from fats. Animal products were limited to 1 portion of low-fat yogurt per day. The diabetic diet provided $50 \%$ of $\mathrm{E}$ from carbohydrates, $20 \%$ from proteins and less than $30 \%$ from fats, where $<7 \%$ of $E$ came from SFA. The diet contained less than $200 \mathrm{mg}$ of cholesterol per day. Both diets were isocaloric, with energy values reduced by $500 \mathrm{kcal}$ per day in relation to the total metabolism. In both groups, the diets were supplemented with $50 \mu \mathrm{g}$ per day of vitamin B12. From the $13^{\text {th }}$ week of the study, the nutritional strategies were combined with personalized exercises. After the end of the study, a significantly higher level of insulin sensitivity was assessed among the people following a vegetarian diet. In addition, a greater decrease in visceral and subcutaneous fat, a significant decrease in inflammation and decreased leptin levels were observed in this group.

Women with IR often have coexisting polycystic ovary syndrome (PCOS). In a study involving 60 such patients, it was estimated that 2 weeks on a reduced-calorie DASH diet (about 350-700 kcal below the total daily energy expenditure), providing 16-18\% of energy from proteins, $30 \%$ from fats and 52-55\% from carbohydrates, contributed to a higher reduction in fasting insulin levels, a higher reduction in HOMA-IR index values and a higher increase in insulin sensitivity levels compared to diets with a similar distribution of macroelements but obtained from other food sources. In addition, following the DASH diet was associated with an improvement in sex hormone levels. ${ }^{45}$

Similar results were obtained by Asemi et al. ${ }^{46}$ In a group of 48 women with PCOS, following the DASH diet was associated with a significant reduction in insulin levels and HOMA-IR index values compared to a diet with the same distribution of macroelements, but obtained from other food sources. For 8 weeks, the subjects in both the study group and the control group followed diets which provided $18 \%$ of $\mathrm{E}$ from protein, $52 \%$ from carbohydrates and $30 \%$ from fats. The nutrition plans differed only in the number of portions of individual food products. The study group (the DASH diet) consumed less than 2,400 mg of sodium per day. Their diet was rich in vegetables, fruits, whole grains, and low-fat dairy products.

\section{Low-carbohydrate diets and IR}

Apart from modification of the quality and quantity of carbohydrates in the diets of insulin-resistant individuals, many researchers also assess the impact of the quantity and quality of dietary fats on glucose and insulin metabolism.

In a study involving 513 Japanese aged 35-70 years, based on data from FFQs containing 46 products and an assessment of dietary habits, it was found that diets similar to Western diets (i.e., with high content of fat and meat as well as a lot of fried meals) were significantly associated with high and abnormal HOMA-IR index values. ${ }^{47}$
In an 8-week study looking at PCOS women with excessive body mass, it was noted that adhering to a low-starch diet with very low dairy consumption was associated with a significant improvement in fasting insulin levels (52\%), insulin levels $2 \mathrm{~h}$ after an oral glucose tolerance test (37\%) and HOMA-IR index values (51\%, i.e., $3.9 \pm 1.5$ vs $1.9 \pm 0.9$ ) compared to the results from before the dietary intervention. The diet includes low-starch vegetables, low-sugar fruits, lean meat, fish, sea foods, eggs, avocados, olives, nuts, seeds, olive oil, and coconut oil. In addition, approx. $30 \mathrm{~g}$ of hard cheese and $180 \mathrm{~mL}$ of red wine per day were permitted; the patients consumed unlimited amounts of the other permitted ingredients. Compliance with the diet was checked 3 times during the study using a 3-day food intake record. The average nutrient content in the diet was approx. 1,422 $\pm 199 \mathrm{kcal}, 72.1 \pm 16.5 \mathrm{~g}$ fat, including $31.9 \pm 9.1 \mathrm{~g}$ monounsaturated FA, $15.8 \pm 6.8$ g polyunsaturated FA, $19.5 \pm 7.8$ g SFA, $94.3 \pm 22.8$ g carbohydrates, and $98 \pm 25.1$ g protein. ${ }^{48}$

However, in another study involving healthy people aged $40-75$ years with BMI values of $25-35 \mathrm{~kg} / \mathrm{m}^{2}$, it was observed that even 1 day on a diet that provides $80 \%$ of $\mathrm{E}$ from fats, $15 \%$ from carbohydrates and $5 \%$ from protein, where $63 \%$ of fats come from SFA (42\% palmitic acid), $29 \%$ from monounsaturated FA (oleic acid from canola oil) and 4\% from polyunsaturated FA, contributed to an increase in whole-body IR in people with normal and impaired glucose tolerance. A decrease in insulin sensitivity was also observed after a single breakfast rich in SFA. ${ }^{49}$

Similar results were reported by Clayton et al., ${ }^{50}$ who assessed the relationship between breakfast composition and changes in insulin sensitivity over a period of 12 weeks. The subjects, who were divided into 2 groups, consumed isocaloric breakfasts consisting of 2 eggs and extras (43\% carbohydrates, $25 \%$ protein, $32 \%$ fats) or a bagel (68\% carbohydrates, $17 \%$ protein, $15 \%$ fats). Up to week 6 , significantly higher insulin sensitivity was observed in the group consuming eggs. However, these values returned to the original state in week 12, when no significant differences were found in fasting insulin and fasting glucose levels between the study groups. The results of the study do not confirm the positive effect of a protein-fat breakfast.

Fat intake may affect epigenetic changes in adipose tissue. It has been observed that adding to the diet $750 \mathrm{kcal}$ from palm oil or refined sunflower oil per day over a period of 7 weeks induces genetic changes in adipose tissue (for example $M c 2 R$ gene). Excessive intake of PUFA from sunflower oli was associated with changes in FTO gene expression and insulin receptor gene expression that are connected with IR. ${ }^{51}$

A meta-analysis of 19 studies showed that replacing $5 \%$ of the energy derived from carbohydrates with SFA or polyunsaturated FA was associated with reductions in fasting insulin levels, by $1.1 \mathrm{pmol} / \mathrm{L}$ and $1.6 \mathrm{pmol} / \mathrm{L}$, respectively. Replacing the energy with monounsaturated FA did not show such an effect. However, introducing 
monounsaturated FA instead of SFA or carbohydrates was associated with lower insulin levels $2 \mathrm{~h}$ after an oral glucose tolerance test. In addition, replacing $5 \%$ of the energy derived from carbohydrates or SFA with energy from polyunsaturated FA resulted in a reduction of HOMA-IR index values by $3.4 \%$ and $4.1 \%$, respectively. ${ }^{52}$

\section{Diets modifying the quantity and quality of carbohydrates and IR}

Many researchers calculate the appropriate amount of carbohydrates for people with IR on the basis of glycemic indices and loads. ${ }^{53-55}$ The majority emphasize the importance of the qualitative composition of carbohydrates in products.

A 12 -week study examined 20 people with the same level of physical activity ( 5 times a week, approx. $1 \mathrm{~h}$, up to $85 \%$ of maximum heart rate) who followed high- or lowglycemic-index diets. No significant differences in insulin sensitivity levels (measured with oral glucose tolerance tests and muscle biopsies) were found between the study groups. ${ }^{53}$

Another study assessed that following a diet with a low glycemic index and low glycemic load (approx. 34 and $<125$, respectively) had a beneficial effect on fasting glucose levels and was associated with a $4 \%$ decrease in insulin-like growth factor-1 (IGF-1) levels, compared to a diet with a glycemic index of approx. 78 and a glycemic load $>250$. In addition, it was noted that the consumption of a lowglycemic-index breakfast significantly correlated with a better insulin response compared to a high-glycemicindex meal both among people with normal body weight and those with excessive body weight. ${ }^{54}$

\section{Daily meal frequency, eating habits and IR}

In addition to the quantity and quality of nutrients, many researchers indicate that daily meal frequency and eating habits are important factors in planning a diet for people with IR.

In a study involving 6 healthy people, Morgan et al. showed that the consumption of $60 \%$ of $E$ at breakfast, $20 \%$ at lunch and $20 \%$ at dinner was significantly associated with better insulin sensitivity compared to the consumption of approx. $20 \%$ of $\mathrm{E}$ at breakfast, $20 \%$ at lunch and $60 \%$ at dinner. In addition, they observed higher insulin sensitivity levels in a group following a low-glycemic-index diet (average glycemic index: 34) compared to a group following high-glycemic-index diet (average glycemic index: 84). Meal frequency, their calorific value and glycemic index all influenced the values of indicators used to diagnose IR. Based on the results of the study it can be suggested that people trying to regulate both glycemia and insulinemia should avoid eating abundant dinners with a high glycemic index. ${ }^{55}$

In a study on the impact of meal frequency on the overall insulin sensitivity in a group of 54 patients with type 2 diabetes, it was found that consuming 2 meals a day, i.e., breakfast between 6 am and 10 am and lunch between $12 \mathrm{am}$ and $4 \mathrm{pm}$, over a period of 12 weeks was associated with higher insulin sensitivity, measured with the oral glucose insulin sensitivity (OGIS) index, compared to the consumption of 6 smaller meals. Both study groups followed the same diet, with energy content reduced by $500 \mathrm{kcal}$, in which $55 \%$ of E came from carbohydrates, 20-25\% from protein and $<30 \%$ from fats ( $<7 \%$ from SFA). In addition, the diet contained less than $200 \mathrm{mg}$ of cholesterol and approx. 30-40 g of dietary fiber. ${ }^{56}$

Similar results were obtained in a study involving 36 people with excessive body weight. It was observed that increased calorie intake in the first half of the day, i.e., 70\% of $\mathrm{E}$ coming from the first 3 meals (breakfast, a morning snack and lunch), was associated with lower HOMA-IR index values, greater weight loss, and greater reduction of waist circumference and body fat percentage compared to a diet providing $55 \%$ of $\mathrm{E}$ in the first half of the day. Regardless of daily meal frequency, the subjects of both groups followed an isocaloric Mediterranean diet with energy content reduced by $600 \mathrm{kcal}$ for a period of 3 months. The diet provided 55-59\% of E from carbohydrates, 25$30 \%$ from fat and approx. 15-16\% from proteins. The subjects who obtained better results consumed approx. 25\% of $\mathrm{E}$ at breakfast, $10 \%$ in a morning snack, 35\% at lunch, $10 \%$ in an afternoon snack, and $20 \%$ at dinner, while the other group consumed $15 \%$ of $\mathrm{E}$ at breakfast, $5 \%$ in a morning snack, $35 \%$ at lunch, $15 \%$ in an afternoon snack, and 30\% at supper. ${ }^{57}$

Similar results were obtained in a study involving 60 thin PCOS women who followed an isocaloric diet $(1,800 \mathrm{kcal}$, approx. 124 g carbohydrates, $191 \mathrm{~g}$ protein and $62 \mathrm{~g}$ fat) with different calorie distribution throughout the day for 3 months. It was found that the consumption of $980 \mathrm{kcal}$ for breakfast, $640 \mathrm{kcal}$ for lunch and $190 \mathrm{kcal}$ for dinner was associated with an $8 \%$ reduction in fasting glucose levels and a 53\% reduction in fasting insulin levels compared to the results of subjects who consumed $190 \mathrm{kcal}$ for breakfast, $640 \mathrm{kcal}$ for lunch and $980 \mathrm{kcal}$ for dinner. In addition, it was noted that the diet with the high-calorie breakfast was associated with a $20 \%$ decrease in the area under the glucose curve and a 49\% decrease in the area under the insulin curve after oral glucose tolerance tests. What is more, the diet with the high-calorie breakfast was associated with 56\% lower HOMA-IR values and 35\% lower HOMA of $\beta$-cell function (HOMA-B) values. It was also associated with the regulation of sex hormone levels and increased rates of ovulation..$^{58}$

A study involving 956 people investigated how daily meal frequency, the manner of eating and the socio-cultural context were related to the occurrence of IR. With 
the use of the Mealtime Habits Quality (MHQ) scale, the authors assessed 4 eating habits: the amount of time available for a meal, distractions while eating, the place and company during the meal, as well as independence in choosing the portion and composition of the meal. The individuals with higher scores on the MHQ scale were those who ate without rush, had enough time for a meal, ate without distractions (reading, working, watching TV), chose the quantity and quality of products themselves, and ate most meals at home, often with their families. Over a period of 8 years, it was observed that the incidence of IR (HOMA-IR > 3.2) was lower in this group compared to people with low MHQ scores (1.2\% vs $12.5 \%)$. People with low MHQ scores had an approx. 12 times higher risk of IR, while those with an average score had about 5 times higher risk of IR compared to the group with high MHQ scores. ${ }^{59}$

Studies concerning meal frequency during the day and their calorific value have provided interesting observations in small research groups. Confirmation in larger randomized trials is needed in order to use the findings as a basis for formulating a diet for insulin-resistant people.

\section{Summary}

Based on the available literature regarding the relationship between diet and IR it seems that reduction of body mass resulting from following a diet with calorific value reduced by approx. 500-600 kcal in relation to total daily energy expenditure should be the basic dietary recommendation for people with impaired glucose/insulin homeostasis.

Observations from recent studies do not indicate a necessity to exclude dairy products from the diet of insulinresistant people. It has been suggested that the consumption of calcium-rich foods, such as dairy and milk products, may have beneficial effects on insulin sensitivity.

The quantity and quality of carbohydrates is a key element in the diet of people with IR. Various authors indicate the merits of reducing the intake of simple sugars, especially those derived from sweet drinks, sweets and excessive fruit juices (more than 2 cups of juice as a equivalent of 2 fruit portions - according to USDA). Many of them have demonstrated the beneficial effect of eating slowly digested complex carbohydrates rich in dietary fiber. The diet of people with IR should be rich in whole grains and significant amounts of non-starchy vegetables and fruit. Insulin-resistant patients should consume carbohydrate products with a low glycemic index to improve insulin sensitivity.

Studies involving insulin-resistant people confirm the beneficial effects of the DASH diet and Mediterranean diet. In contrast, the benefits of low- and very-low-carbohydrate diets require confirmation in long-term studies with large study groups. However, it seems that less restrictive limitation of carbohydrates (up to approx. 40\%) through the reduction of simple sugars and starch intake may have a beneficial effect on insulin homeostasis.

Studies evaluating the relationship between diet and IR provide interesting observations and possible dietary recommendations. However, due to study limitations, their utility for insulin-resistant patients requires confirmation in studies with larger cohorts.

Studies of the influence of the quality of consumed fats on IR showed that a high intake of SFA has a negative impact on IR. However, it has been observed that increased consumption of monounsaturated FA and polyunsaturated FA (mainly omega-3) has a positive effect on the parameters associated with IR.

In addition, it has been noted that moderate amounts of alcohol as part of a balanced low-calorie diet did not have a negative impact on insulin-resistant people. Similar observations have been made with regard to chocolate. It seems that moderate amounts of these products can be a positive element that increases patients' compliance with nutritional recommendations.

The effectiveness of dietary recommendations among people with IR may depend not only on the quality of the products, but also on daily meal frequency and meal energy content. It has been suggested that increased calorie intake in the first half of the day, with particular emphasis on an abundant, low-glycemic-index breakfast containing complex carbohydrates, contributes to reductions in IR. In addition, many researchers recommend slow and mindful eating as part of a healthy diet for insulinresistant people.

\section{References}

1. Ioannou GN, Bryson CL, Boyko EJ. Prevalence and trends of insulin resistance, impaired fasting glucose and diabetes. J Diabetes Complications. 2007;21(6):363-370.

2. Grzesiuk W, Szydlarska D, Jóźwik K. Insulinooporność w endokrynopatiach. Endokrynol Otył Zab Przem Mat. 2008;4(1):38-44.

3. Wang $X$, Xian T, Jia X, et al. A cross-sectional study on the associations of insulin resistance with sex hormone, abnormal lipid metabolism in T2DM and IGT patients. Medicine (Baltimore). 2017;96(26):e7378.

4. Park MH, Kim DH, Lee EK, et al. Age-related inflammation and insulin resistance: A review of their intricate interdependency. Arch Pharm Res. 2014;37(12):1507-1514.

5. Geer EB, Shen W. Gender differences in insulin resistance, body composition, and energy balance. Gend Med. 2009;6(Suppl 1):60-75.

6. Kitamoto A, Kitamoto T, So R, et al. ADIPOQ polymorphisms are associated with insulin resistance in Japanese women. Endocr J. 2015; 62(6):513-521.

7. Shakil-Ur-Rehman S, Karimi H, Gillani SA. Effects of supervised structured aerobic exercise training program on fasting blood glucose level, plasma insulin level, glycemic control, and insulin resistance in type 2 diabetes mellitus. Pak J Med Sci. 2017;33(3):576-580.

8. Yan YX, Xiao HB, Wang SS, et al. Investigation of the relationship between chronic stress and insulin resistance in a Chinese population. J Epidemiol. 2016;26(7):355-360.

9. Tilg $\mathrm{H}$, Moschen AR. Inflammatory mechanisms in the regulation of insulin resistance. Mol Med. 2008;14(3-4):222-231.

10. Aldhoon-Hainerova I, Zamrazilova H, Dusatkova L, et al. Glucose homeostasis and insulin resistance: Prevalence, gender differences and predictors in adolescents. Diabetol Metab Syndr. 2014;6:100. doi:10.1186/1758-5996-6-100 
11. Do HD, Lohsoonthorn V, Jiamjarasrangsi W, Lertmaharit S, Williams MA. Prevalence of insulin resistance and its relationship with cardiovascular disease risk factors among Thai adults over 35 years old. Diabetes Res Clin Pract. 2010;89(3):303-308.

12. Eglit T, Rajasalu T, Lember M. Metabolic syndrome in Estonia: Prevalence and associations with insulin resistance. Int J Endocrinol. 2012; 2012:951672. doi:10.1155/2012/951672

13. Verhagen SN, Wassink AMJ, van der Graaf Y, Gorter PM, Visseren FL; SMART Study Group. Insulin resistance increases the occurrence of new cardiovascular events in patients with manifest arterial disease without known diabetes. The SMART study. Cardiovasc Diabetol. 2011;10:100. doi:10.1186/1475-2840-10-100

14. Otake T, Fukumoto J, Abe M, et al. Linking lifestyle factors and insulin resistance, based on fasting plasma insulin and HOMA-IR in middleaged Japanese men: A cross-sectional study. Scand J Clin Lab Invest. 2014;74(6):536-545.

15. Gunji T, Matsuhashi N, Sato H, et al. Alcohol consumption is inversely correlated with insulin resistance, independent of metabolic syndrome factors and fatty liver diseases. J Clin Gastroenterol. 2011;45(9): 808-813.

16. Schrieks IC, Heil AL, Hendriks HF, Mukamal KJ, Beulens JW. The effect of alcohol consumption on insulin sensitivity and glycemic status: A systematic review and meta-analysis of intervention studies. Diabetes Care. 2015;38(4):723-732.

17. Skropanic D, Fufaa G, Cai B. The association between changes in insulin sensitivity and consumption of tobacco and alcohol in young adults: Ordinal logistic regression approach. Cureus. 2016;8(12):e942.

18. Funayama T, Tamura Y, Takeno K, et al. Effects of alcohol abstinence on glucose metabolism in Japanese men with elevated fasting glucose: A pilot study. Sci Rep. 2017;7:40277.

19. Gunnerud U, Holst JJ, Östman E, Björck I. The glycemic, insulinemic and plasma amino acid responses to equi-carbohydrate milk meals: A pilot-study of bovine and human milk. Nutr J. 2012:83. doi:10.1186/ 1475-2891-11-83

20. Hashemipour S, Esmailzadehha N, Mohammadzadeh M, Ziaee A. Association of meat and dairy consumption with normal weight metabolic obesity in men: The Qazvin Metabolic Disease Study. Eat Weight Disord. 2016;21(3):419-425.

21. Rideout TC, Marinangeli CP, Martin H, Browne RW, Rempel CB. Consumption of low-fat dairy foods for 6 months improves insulin resistance without adversely affecting lipids or bodyweight in healthy adults: A randomized free-living cross-over study. Nutr J. 2013;12:56. doi:10.1186/1475-2891-12-56

22. Hansen CK, Klingenberg L, Larsen LB, Lorenzen JK, Sørensen KV Astrup A. The effect of UHT-processed dairy milk on cardio-metabolic risk factors. Eur J Clin Nutr. 2017;71(12):1463-1466. doi:10.1038/ ejcn.2017.22

23. Turner KM, Keogh JB, Clifton PM. Red meat, dairy, and insulin resistance sensitivity: A randomized crossover intervention study. Am J Clin Nutr. 2015;101(6):1173-1179.

24. Wallace IR, McEvoy CT, Hunter SJ, et al. Dose-response effect of fruit and vegetables on insulin resistance in people at high risk of cardiovascular disease: A randomized controlled trial. Diabetes Care. 2013;36(12):3888-3896.

25. Dhillon PK, Bowen L, Kinra S, et al; Indian Migration Study Group. Legume consumption and its association with fasting glucose, insulin resistance and type 2 diabetes in the Indian Migration Study. Public Health Nutr. 2016;19(16):3017-3026.

26. Menderson M, Benedetti A, Gray-Donald K. Dietary composition and its associations with insulin sensitivity and insulin secretion in youth. Br J Nutr. 2014;111(3):527-534.

27. Yeo R, Yoon Sr, Kim OY. The association between food group consumption patterns and early metabolic syndrome risk in non-diabetic healthy people. Clin Nutr Res. 2017;6(3):172-182.

28. Cavallo DN, Horino M, Mc Carthy WJ. Adult intake of minimally processed fruits and vegetables: Associations with cardiometabolic disease risk factors. J Acad Nutr Diet. 2016;116(9):1387-1394.

29. Wang B, Liu K, Mi M, Wang J. Effect of fruit juice on glucose control and insulin sensitivity in adults: A meta-analysis of 12 randomized controlled trials. PLoS One. 2014;9(4):e95323.
30. Simpson EJ, Mendis B, Macdonald IA. Orange juice consumption and its effect on blood lipid profile and indices of the metabolic syndrome: A randomised, controlled trial in an at-risk population. Food Funct. 2016;7(4):1884-1891.

31. Sese MA, Jimenez-Pavon D, Gilbert CC, et al; HELENA Study Group. Eating behavior, insulin resistance and cluster of metabolic risk factors in European adolescents. The HELENA Study. Appetite. 2012; 59(1):140-147.

32. Silveira JQ, Dourado GK, Cesar TB. Red-fleshed sweet orange juice improves the risk factors for metabolic syndrome. Int J Food Sci Nutr. 2015;66(7):830-836.

33. Heikkilä HM, Krachler B, Rauramaa R, Schwab US. Diet, insulin secretion and insulin sensitivity - the Dose-Responses to Exercise Training (DR's EXTRA) Study (ISRCTN45977199). Br J Nutr. 2014;112(9):15301541. doi:10.1017/S0007114514002426

34. Damsgaard C, Biltoft-Jensen A, Tetens I, et al. Whole-grain intake, reflected by dietary records and biomarkers, is inversely associated with circulating insulin and other cardiometabolic markers in 8to 11-year-old children. J Nutr. 2017;147(5):816-824. doi:10.3945/jn. 116.244624

35. Vinoy S, Meynier A, Goux A, et al. The effect of a breakfast rich in slowly digestible starch on glucose metabolism: A statistical metaanalysis of randomized controlled trials. Nutrients. 2017;9(4):318.

36. MacNeil S, Rebry RM, Tetlow IJ, Emes MJ, McKeown B, Graham TE. Resistant starch intake at breakfast affects postprandial responses in type 2 diabetics and enhances the glucose-dependent insulinotropic polypeptide-insulin relationship following a second meal. Appl Physiol Nutr Metab. 2013;36(12):1187-1195.

37. Ma J, Jacques PF, Meigis JB, et al. Sugar-sweetened beverage but not diet soda consumption is positively associated with progression of insulin resistance and prediabetes. J Nutr. 2016;146(12):2544-2550.

38. O'Connor L, Imamura F, Brage S, Griffin SJ, Wareham NJ, Forouhi NG. Intakes and sources of dietary sugars and their association with metabolic and inflammatory markers. Clin Nutr. 2018;37(4):1313-1322. doi:10.1016/j.clnu.2017.05.030

39. McNaughton SA, Mishra GD, Brunner EJ. Dietary patterns, insulin resistance, and incidence of type 2 diabetes in the Whitehall II Study. Diabetes Care. 2008;31(7):1343-1348.

40. Karatzi K, Maschonis G, Barouti AA, Lionis C, Chrousos GP, Manios Y; Healthy Growth Study Group. Dietary patterns and breakfast consumption in relation to insulin resistance in children. The Healthy Growth Study. Publ Heath Nutr. 2014;17(12):2790-2797. doi:10.1017/ S1368980013003327

41. Alkerwi A, Sauvageot N, Crichton GE, Elias MF, Stranges S. Daily chocolate consumption is inversely associated with insulin resistance and liver enzymes in the Observation of Cardiovascular Risk Factors in Luxembourg study. Br J Nutr. 2016;115(9):1661-1668.

42. Park YM, Zhang J, Steck SE, et al. Obesity mediates the association between Mediterranean diet consumption and insulin resistance and inflammation in US adults. J Nutr. 2017;147(4):563-571.

43. Mattei J, Sotos-Prieto M, Bigornia SJ, Noel SE, Tucker KL. The Mediterranean diet score is more strongly associated with favorable cardiometabolic risk factors over 2 years than other diet quality indexes in Puerto Rican adults. J Nutr. 2017;147(4):661-669.

44. Kahleova $\mathrm{H}$, Matoulek M, Malinska $\mathrm{H}$, et al. Vegetarian diet improves insulin resistance and oxidative stress markers more than conventional diet in subjects with type 2 diabetes. Diabet Med. 2011;28(5): 549-559.

45. Foroozanfard F, Raflei H, Samimi M, et al. The effects of dietary approaches to stop hypertension diet on weight loss, anti-Müllerian hormone and metabolic profiles in women with polycystic ovary syndrome: A randomized clinical trial. Clin Endocrinol (Oxf). 2017;87(1): 51-58.

46. Asemi Z, Esmaillzadeh A. DASH diet, insulin resistance, and serum hs-CRP in polycystic ovary syndrome: A randomized controlled clinical trial. Horm Metab Res. 2015;47(3):232-238.

47. Arisawa $\mathrm{K}$, Uemura $\mathrm{H}$, Yamaguchi $\mathrm{M}$, et al. Associations of dietary patterns with metabolic syndrome and insulin resistance: A crosssectional study in a Japanese population. J Med Invest. 2014;61(3-4): 333-344. 
48. Phy JL, Pohlmeier AM, Cooper JA, et al. Low starch/low dairy diet results in successful treatment of obesity and co-morbidities linked to polycystic ovary syndrome (PCOS). J Obes Weight Loss Ther. 2015; 5(2):259.

49. Koska J, Ozias MK, Deer J, et al. A human model of dietary saturated fatty acid induced insulin resistance. Metabolism. 2016;65(11):16211628.

50. Clayton ZS, Scholar KR, Shelechi M, et al. Influence of resistance training combined with daily consumption of an egg-based or bagel-based breakfast on risk factors for chronic diseases in healthy untrained individuals. J Am Coll Nutr. 2015;34(2):113-119.

51. Perfilyev A, Dahlman I, Gillberg L, et al. Impact of polyunsaturated and saturated fat overfeeding on the DNA-methylation pattern in human adipose tissue: A randomized controlled trial. Am J Clin Nutr. 2017;105(4):991-1000. doi:10.3945/ajcn.116.143164

52. Imamura F, Micha $\mathrm{R}, \mathrm{Wu} \mathrm{JH}$, et al. Effects of saturated fat, polyunsaturated fat, monounsaturated fat, and carbohydrate on glucoseinsulin homeostasis: A systematic review and meta-analysis of randomised controlled feeding trials. PLoS Med. 2016;13(7):e1002087.

53. Muya A, Haus JM, Solomon TP, et al. Exercise training-induced improvement in skeletal muscle PGC-1a-mediated fat metabolism is independent of dietary glycemic index. Obesity (Silver Spring). 2017; 25(4):7212-7229.
54. Runchey SS, Pollak MN, Valsta LM, et al. Glycemic load effect on fasting and post-prandial serum glucose, insulin, IGF-1 and IGFBP-3 in a randomized, controlled feeding study. Eur J Clin Nutr. 2012;66(10): 1146-1152.

55. Morgan LM, Shi JW, Hampton SM, Frost G. Effect of meal timing and glycemic index on glucose control and insulin secretion in healthy volunteers. Br J Nutr. 2012;108(7):1286-1291.

56. Kahleova $\mathrm{H}$, Belinova $\mathrm{L}$, Malinska $\mathrm{H}$, et al. Eating two larger meals a day (breakfast and lunch) is more effective than six smaller meals in a reduced energy regimen for patients with type 2 diabetes: A randomized crossover study. Diabetologia. 2014;57(8):1552-1560.

57. Lombardo $M$, Bellia $A$, Padua $E$, et al. Morning meal more efficient for fat loss in a 3-month lifestyle intervention. J Am Coll Nutr. 2014;33(3): 198-205.

58. Jakubowicz D, Barnea M, Wainstein J, Froy O. Effects of calorific intake timing on insulin resistance and hyperandrogenism in lean women with polycystic ovary syndrome. Clin Sci (Lond). 2013;125(9):423-432.

59. Mendez-Hernandez P, Dosamantes-Carrasco LD, Siani C, et al. Mealtime habits and risk of developing the metabolic syndrome or insulin resistance among Mexican adults. Br J Nutr. 2016;11(10):1824-1833. 